\title{
Elastic Constants of Synthetic Single Crystal Corundum
}

\author{
Wayne E. Tefft*
}

\author{
(March 10, 1966) \\ The elastic constants of synthetic single crystal corundum (aluminum oxide) were calculated from \\ 0 to $900{ }^{\circ} \mathrm{K}$ from data obtained by a resonance technique from 80 to $900{ }^{\circ} \mathrm{K}$.
}

Key Words: Aluminum oxide, corundum, elastic constants, shear modulus, Young's modulus.

\section{Introduction}

Two previous papers $[1,2]^{1}$ have presented respectively, the room temperature values of the elastic constants of synthetic single crystal corundum [1], and the temperature dependence of Young's modulus for certain crystallographic directions. In the present paper we present results of an extension of that work to include measurements of the temperature dependence of the torsional modulus for certain crystallographic directions, and a calculation from all these measurements of the complete set of elastic compliances from 0 to $900{ }^{\circ} \mathrm{K}$. There has been a lapse of several years between the time of performance of this work and its submission for publication, and another paper on the subject [3] has appeared in the meantime. However, the present results cover a wider temperature range and are believed to be more accurate than those previously published.

This work was undertaken for several reasons. First, a knowledge of elastic constants is desirable for an understanding of the interatomic forces in solids, and most useful in this respect are the values of these constants at absolute zero. Second, the variation of elastic constants with temperature is one important test of theories of lattice dynamics.

The availability of different kinds of data on the same material is also of considerable theoretical importance, because of the interrelations between various thermodynamic properties. Since corundum is of technological importance and good single crystal specimens are readily available, very accurate measurements of its specific heat [4] and thermal expansion [5] have already been made. Thus, elastic constant data are desirable to permit a test of thermodynamic relations in which elastic constants appear [5].

Finally, the results presented here may be considered as an example of the precision attainable with the resonance method of elastic constant measurements in its most refined form. It is believed by this author that none of the other techniques in current use is more accurate than the one used here, and these data are presented to support this view.

*Present Address: Xerox Corporation, Solid State Research Department, Rochester, N.Y. ${ }^{1}$ Figures in brackets indicate the literature references at the end of this paper.

\section{Experimental Procedure}

The determination of the elastic constants of corundum at room temperature, and the temperature dependence of Young's modulus of seven sapphire rods of various orientations, have already been reported $[1,2]$. The additional data taken for this experiment consisted of measurements of the temperature dependence of the torsional resonance frequencies of six of the seven rods from 80 to $875^{\circ} \mathrm{K}$. About 40 measurements of torsional frequency were made on each rod. Most of the experimental apparatus was identical in these three studies, and it will be assumed that the reader is familiar with the first two papers.

The torsional resonance frequencies of the original rods were of the order of $20 \mathrm{kHz}$, which was too high to be excited and detected in the cryostat, and which would have been difficult to measure in the furnace. In order to overcome this problem, the diameter of each rod was reduced by about 50 percent over approximately the middle two-thirds of its length. This was equivalent to attaching loading disks to the ends, in that the resonance frequency was thereby reduced to about one-third of its original value, but this procedure was preferable to that of attaching loading disks because it did not introduce the problem of finding a bonding material suitable for attaching loading disks and unaffected by the wide range of temperatures used. The resultant shape made it impossible to calculate absolute values of the torsional modulus from the resonance frequencies, but since the values at room temperature were already known, only changes with temperature were needed, and the temperature dependence of the modulus frequency relationship was assumed to be the same as for perfect cylinders. This assumption undoubtedly introduced some errors, but these errors are believed to be very small because they would enter through the effect of thermal expansion on small correction factors.

Torsional vibrations were excited and detected in the furnace as previously described [1] for room temperature, except that glass fiber instead of thread was used for the suspension. In order to make measurements at low temperatures, small flats were ground at each end of the specimen, and very small 
magnets attached to these. The specimen was then suspended in a horizontal position by two threads in such a way that each magnet was perpendicular to the field of a nearby coil. When an alternating current was passed through one coil, a net torque resulted, and the corresponding vibrations of the rod induced currents in the other coil, which was used to detect resonance. In both high- and low-temperature measurements, the precision of the frequency determinations was of the order of one part in 30,000 , although temperature gradients and systematic errors such as changing hardness of bond between magnet and specimen and condensation of ice at low temperature, and gradual crystallization of the glass fibers used for suspension at high temperatures, probably reduced the overall accuracy to about one part in $10^{4}$.

The relation between resonance frequency, $f$, density, $\rho$, length, $l$, and torsional modulus, $G$, of an isotropic cylinder is [1]

$$
G=4 \rho l^{2} f^{2} .
$$

Thus, if our specimens had been isotropic cylinders, the torsional modulus, $G$, at any temperature, $T$, would be related to the torsional modulus at room temperature, $G_{R}$, by

$$
G=G_{R}\left(\frac{\rho}{\rho_{R}}\right)\left(\frac{l}{l_{R}}\right)^{2}\left(\frac{f}{f_{R}}\right)^{2}
$$

Now if the specimens had been perfect cylinders at room temperature, the anisotropic thermal expansion would have caused their cross sections to become elliptical at any other temperature, because for a crystal of trigonal symmetry, the thermal expansion (fractional change in length) in any direction is given by [5]

$$
\epsilon(\theta)=\epsilon_{1} \cos ^{2} \theta+\epsilon_{2} \sin ^{2} \theta
$$

where $\epsilon_{1}$ and $\epsilon_{2}$ are, respectively, the thermal expansions parallel and perpendicular to the crystallographic $c$-axis, and $\theta$ is the angle between the $c$-axis and the direction under consideration. Thus, the circular cylinder of length $l_{R}$ and radius $r$ at room temperature becomes an elliptical cylinder of length $l$ and semiaxes $a$ and $b$ at other temperatures. If $a$ is chosen to be perpendicular to both the rod axis and the $c$-axis, and $b$ is in the plane formed by the rod axis and the $c$-axis, then these quantities are given by:

$$
\begin{aligned}
& l=l_{R}\left(1+\epsilon_{1} \cos ^{2} \theta+\epsilon_{2} \sin ^{2} \theta\right) \\
& a=r\left(1+\epsilon_{2}\right) \\
& b=r\left(1+\epsilon_{1} \sin ^{2} \theta+\epsilon_{2} \cos ^{2} \theta\right) .
\end{aligned}
$$

The density is $\frac{m}{\pi a b l}$, so that the equation for $G$ becomes

$$
\begin{aligned}
G=G_{R} \frac{r^{2} l_{R}}{a b l} \frac{l^{2}}{l_{R}^{2}} \frac{f^{2}}{f_{R}^{2}}=G_{R} \frac{r^{2} l}{a b l_{R}}\left(\frac{f}{f_{R}}\right)^{2} \\
=G_{R} \frac{1+\epsilon_{1} \cos ^{2} \theta+\epsilon_{2} \sin ^{2} \theta}{\left(1+\epsilon_{2}\right)\left(1+\epsilon_{1} \sin ^{2} \theta+\epsilon_{2} \cos ^{2} \theta\right)}\left(\frac{f}{f_{R}}\right)^{2}
\end{aligned}
$$

which reduces approximately to

$$
G \approx G_{R}\left(\frac{f}{f_{R}}\right)^{2}\left[1-\epsilon_{1}+2\left(\epsilon_{1}-\epsilon_{2}\right) \cos ^{2} \theta+\epsilon_{1}^{2}\right]
$$

where the square and higher powers of the difference $\left(\epsilon_{1}-\epsilon_{2}\right)$, and the cube and higher powers of $\epsilon_{1}$ and $\epsilon_{2}$ have been neglected.

Now, it is true that eq (1) is correct only for a circular cylinder, and that it should be modified for the elliptical shape caused by anisotropic thermal expansion. However, it may be shown from the equations of static torsion of elliptical cylinders that the largest correction term is proportional to $\left(\frac{a-b}{r}\right)^{2}$ which is
always very small.

A more serious question is what modification is necessary because of the reduction in diameter over a portion of the length. There seems to be no very rigorous argument here, but one can say qualitatively that since the correction for thermal expansion is quite small, no appreciable error is likely to be introduced because this correction is not made exactly as it should be.

\section{Analysis of Data}

The systematic errors in the compliances are believed to be smaller than the error introduced by random error in the orientations. In order to achieve a higher degree of accuracy, the amount of data taken was considerably larger than the minimum needed to determine the six independent elastic constants of sapphire. An extensive statistical analysis of these data was made to determine the best values of these constants, and their variation with temperature.

In the original room temperature work, Young's modulus and the torsional modulus were measured on 29 different specimens, and a standard least-squares routine was then used to evaluate the six independent constants from this set of 58 measurements. It was noted, however, that the differences between measured and calculated values of $E$ and $G$ were due primarily to errors in orientation measurements, rather than to errors in the measured values of $E$ and $G$ which is implicitly assumed in the standard least-squares calculation. In order to take this into account, weights were determined by a method described by Deming [6]. 
After having redetermined the elastic constants at room temperature, and having measured both $E$ and $G$ for several orientations as a function of temperature, the problem arose as to how best to use this information to estimate the temperature dependence of the elastic constants. If measurements on all specimens had been made at identical temperatures, say $50^{\circ}$ intervals, then one might simply have carried out the weighted least-squares routine at each of these temperatures, and thereby obtained an adequate representation of the temperature dependence of the elastic constants. This, however, would have involved an enormous amount of experimental work, and would not have significantly improved the accuracy of the results, basically because changes in the constants could be measured with better precision than the absolute values of the constants, and therefore a smaller number of measurements was needed.

It was pointed out in reference 2 that an empirical equation had been found to accurately represent the variation of Young's modulus with temperature. While fitting the experimental data to this equation by means of a least-square routine on an automatic computer, calculations were also made of the values of Young's modulus at $50^{\circ}$ intervals from 0 to $900{ }^{\circ} \mathrm{K}$. An attempt was made to fit the torsional modulus variation to the same form of equation. In this case, although the fit was reasonably good, the deviations appeared to be systematic, indicating that the equation did not represent the data within the experimental precision. Therefore, a plot of deviations versus temperature was made for each specimen, and corrections ranging up to about 0.04 percent were made for the calculated values of $G$ at the same $50^{\circ}$ temperature intervals.

A further correction was necessitated because the temperature dependence was measured for fewer specimens than were used in the room temperature measurements. It was first thought that an adequate correction would be to adjust the base (room temperature) values of $E$ and $G$ for each specimen so that they agreed with the calculated values in the room temperature curve fit. However, it was noted that the elastic anisotropy increased rather markedly with temperature, and that orientation errors were introducing appreciable scatter at the highest temperature. In order to partially overcome this difficulty, the orientation angles of two of the specimens (those whose calculated modulus values were most sensitive to orientation errors, and which had the largest deviations at room temperature) were adjusted to give the smallest possible deviations of $E$ and $G$ at room temperature (the deviation of either could be made zero in this manner, but not both simultaneously). The remaining deviations in $E$ and $G$ at room temperature for all specimens were then corrected for, and a least-squares solution for the elastic constants made at $50^{\circ}$ intervals. The final result was that the errors in temperature dependences of the elastic constants were somewhat smaller even at the highest temperature than were their absolute values at room temperature. Therefore, the values quoted at $900{ }^{\circ} \mathrm{K}$ should be in error (in absolute value) by less than twice the errors of absolute value at room temperature. Since measurements were only made down to liquid nitrogen temperature, the errors in the values at absolute zero are somewhat uncertain, but there is reason to believe that they are less in error than the values at $900{ }^{\circ} \mathrm{K}$, although certainly somewhat more in error than the values at room temperature. The reason that these errors are small in spite of the $80^{\circ}$ extrapolation is that nearly all the variation takes place at higher temperature; i.e., the modulus temperature curves are very nearly flat up to about $100{ }^{\circ} \mathrm{K}$.

\section{Results and Summary}

Values of the six elastic compliance constants of corundum in the temperature interval 0 to $900{ }^{\circ} \mathrm{K}$ are given in table 1 . The base values, at $25^{\circ} \mathrm{C}$ and their standard error estimates, are given in the first row.

TABLE.1 The elastic compliances of corundum in units of $10^{-12} \mathrm{~m}^{2} / \mathrm{N}\left(10^{-13} \mathrm{~cm}^{2} / d y n\right)$

The first row lists room temperature values together with their standard deviations. These values were combined with measurements of relative changes with temperature to obtain the values at other temperatures.

\begin{tabular}{c|c|c|c|c|c|c}
\hline \hline $\begin{array}{c}\text { Temper- } \\
\text { ature }\end{array}$ & $s_{11}$ & $s_{33}$ & $s_{44}$ & $s_{12}$ & $s_{13}$ & $s_{14}$ \\
\hline & & & & & & \\
${ }^{\circ} K$ & & & & & & \\
273.2 & 2.353 & 2.168 & 6.940 & -0.716 & -0.368 & 0.491 \\
& \pm 0.002 & \pm 0.002 & \pm 0.008 & \pm .007 & \pm .006 & \pm .005 \\
0 & 2.326 & 2.151 & 6.765 & -.693 & -.362 & .465 \\
100 & 2.326 & 2.152 & 6.776 & -.695 & -.363 & .467 \\
150 & 2.330 & 2.154 & 6.802 & -.698 & -.364 & .471 \\
200 & 2.336 & 2.158 & 6.841 & -.704 & -.365 & .477 \\
250 & 2.344 & 2.163 & 6.889 & -.710 & -.366 & .483 \\
& & & & & & \\
300 & 2.353 & 2.169 & 6.942 & -.716 & -.368 & .491 \\
350 & 2.364 & 2.176 & 7.000 & -.723 & -.369 & .500 \\
400 & 2.375 & 2.183 & 7.061 & -.731 & -.370 & .509 \\
450 & 2.386 & 2.191 & 7.126 & -.740 & -.370 & .519 \\
500 & 2.399 & 2.200 & 7.193 & -.748 & -.371 & .530 \\
& & & & & \\
550 & 2.412 & 2.209 & 7.263 & -.757 & -.372 & .541 \\
600 & 2.425 & 2.218 & 7.334 & -.766 & -.372 & .552 \\
650 & 2.438 & 2.228 & 7.407 & -.775 & -.373 & .564 \\
700 & 2.453 & 2.237 & 7.482 & -.784 & -.373 & .576 \\
750 & 2.467 & 2.247 & 7.559 & -.796 & -.374 & .587 \\
800 & 2.482 & 2.258 & 7.638 & -.808 & -.374 & .600 \\
850 & 2.497 & 2.268 & 7.719 & -.820 & -.374 & .613 \\
900 & 2.512 & 2.279 & 7.803 & -.832 & -.375 & .626 \\
\hline & & & & & & \\
\hline
\end{tabular}

These values were determined from measurements on the same set of 29 specimens used in reference 1 , but the present results are believed to be slightly more accurate because of improved temperature control and use of a weighted least-squares routine.

The values at other temperatures are based on values at $25^{\circ} \mathrm{C}$, and therefore include in their absolute magnitude the errors of the base values. However, the changes with temperature over any small temperature interval were determined more accurately even though based on only seven specimens, because changes in Young's modulus and the torsional modulus could be measured more accurately than the actual values of these moduli. On the other hand, the anisotropy (as estimated, for example, from the variation in Young's modulus with orientation) increased significantly with increasing temperature. Thus, the 
small orientation errors which remained after making the room temperature corrections described earlier would have introduced some error into the temperature dependence of the elastic compliances, even if the moduli had been measured exactly.

The result was that the change in any constant between room temperature and either 0 or $900{ }^{\circ} \mathrm{K}$ was determined with an accuracy approximately equal to the accuracy of that constant at room temperature. One cannot make a more precise statement than this because, while systematic errors are believed to be insignificant in the room temperature results, they may be comparable to the random errors at other temperatures. Furthermore, even random errors, such as would result from misorientation, cannot be estimated very well for the temperature dependence measurements because of the small number of specimens used.

\section{References}

[1] J. B. Wachtman, Jr., W. E. Tefft, D. C. Lam, Jr., and R. P. Stenchfield, Elastic constants of synthetic single crystal corundum at room temperature, J. Res. NBS 64.A (Phys. and Chem.), No. 3, 213-228 (1960).

[2] J. B. Wachtman, Jr., W. E. Tefft, D. C. Lam, Jr., and C. S. Apstein, Exponential temperature dependence of Young's modulus for several oxides, Phys. Rev. 122, 1754-1759 (1961).

[3] P. Jayarama Reddy, Variation with temperature of the elastic compliances of corundum, Acta. Cryst. 16, 335 (1963).

[4] G. T. Furukawa, T. B. Douglas, R. E. McCoskey, and D. C. Ginnings, Thermal properties of aluminum oxide from 0 to $1200{ }^{\circ} \mathrm{K}, \mathrm{J}$. Res. NBS 57, 67 (1956) RP2694.

[5] J. B. Wachtman, Jr., T. G. Scuderi, and G. W. Cleek, Linear thermal expansion of aluminum oxide and thorium oxide from 100 to $1100^{\circ}$ K, J. Am. Ceram. Soc. 45, 319-323 (1962).

[6] W. Edwards Deming, Statistical Adjustment of Data, pp. 152-171 (John Wiley \& Sons, Inc., New York, N.Y., 1943).

(Paper 70A4-403) 